\title{
Nanocrystalline Materials at Equilibrium: A Thermodynamic Review
}

\author{
ARVIND R. KALIDINDI, ${ }^{1}$ TONGJAI CHOOKAJORN, ${ }^{1,2}$ \\ and CHRISTOPHER A. SCHUH ${ }^{1,3}$ \\ 1.-Department of Materials Science and Engineering, Massachusetts Institute of Technology, \\ Cambridge, MA 02139, USA. 2.-National Metal and Materials Technology Center (MTEC), \\ National Science and Technology Development Agency (NSTDA), Pathum Thani 12120, Thailand. \\ 3.—e-mail: schuh@mit.edu
}

The instability of nanocrystalline materials against both grain growth and bulk phase separation is a principal challenge in their production and usage. This article reviews the thermodynamic stabilization of nanocrystalline structures by alloying, where a nanocrystalline state is considered to be stable if the nanostructure has the lowest free energy available to the alloy system, such that it is stable both against grain growth and the formation of bulk second phases. The thermodynamic accessibility of nanocrystalline structures in the alloy phase space introduces configurational degrees of freedom both at the atomic scale of the grain boundary structure and at the mesoscale level of the grains and grain boundary topology, which should be considered when identifying the equilibrium state. This article presents a survey of the kinds of thermodynamic models and simulations that have been developed to search for equilibrium nanocrystalline states. The review emphasizes the utility of Monte Carlo simulations to assess the thermodynamic stability of nanocrystalline states, including methods that have been proposed to account for degrees of freedom at both the atomic and grain scales. Although atomic scale simulations provide detailed segregation energetic information, the topological degrees of freedom in nanoscale polycrystals seem to be more critical considerations in the free energy description for identifying whether a nanocrystalline state is stable, and these are better addressed with mesoscale lattice-based simulation methods. A variety of interesting new nanostructural alloy states awaits further exploration by computational methods.

\section{INTRODUCTION}

The high volume fraction of grain boundaries in nanocrystalline materials pits structural instability against improved performance. The nanoscale grain structure provides these materials with an interesting set of properties such as high strength and wear resistance, but introduces a large driving force for grain growth out of the desired nanocrystalline regime. Alloying has been used to oppose grain growth through at least two different mechanisms: by treating the symptom-grain boundary motionthrough solute drag or second phase pinning ${ }^{1-5}$ or by treating the underlying cause- the excess free energy of the grain boundary, which can be relieved by solute segregation. ${ }^{6-11}$ Whereas slowing grain boundary motion can be sufficient for some applications, the kinetic nature of the drag mechanism often yields a merely transient stability to nanostructures; they eventually prefer to coarsen. Furthermore, solute drag is typically only effective at relatively low homologous temperatures due to the strong temperature dependence of mobility. On the other hand, if alloying can eliminate the driving force for grain growth, then nanocrystalline alloys can be developed that reliably retain their structure for a longer timeat-temperature or at higher temperatures, providing better control over nanostructure, and possibly a wider range of processing routes and applications.

In a seminal paper describing thermodynamic nanostructure stabilization, Weissmüller ${ }^{8}$ argued that if segregation of solute species is energetically 

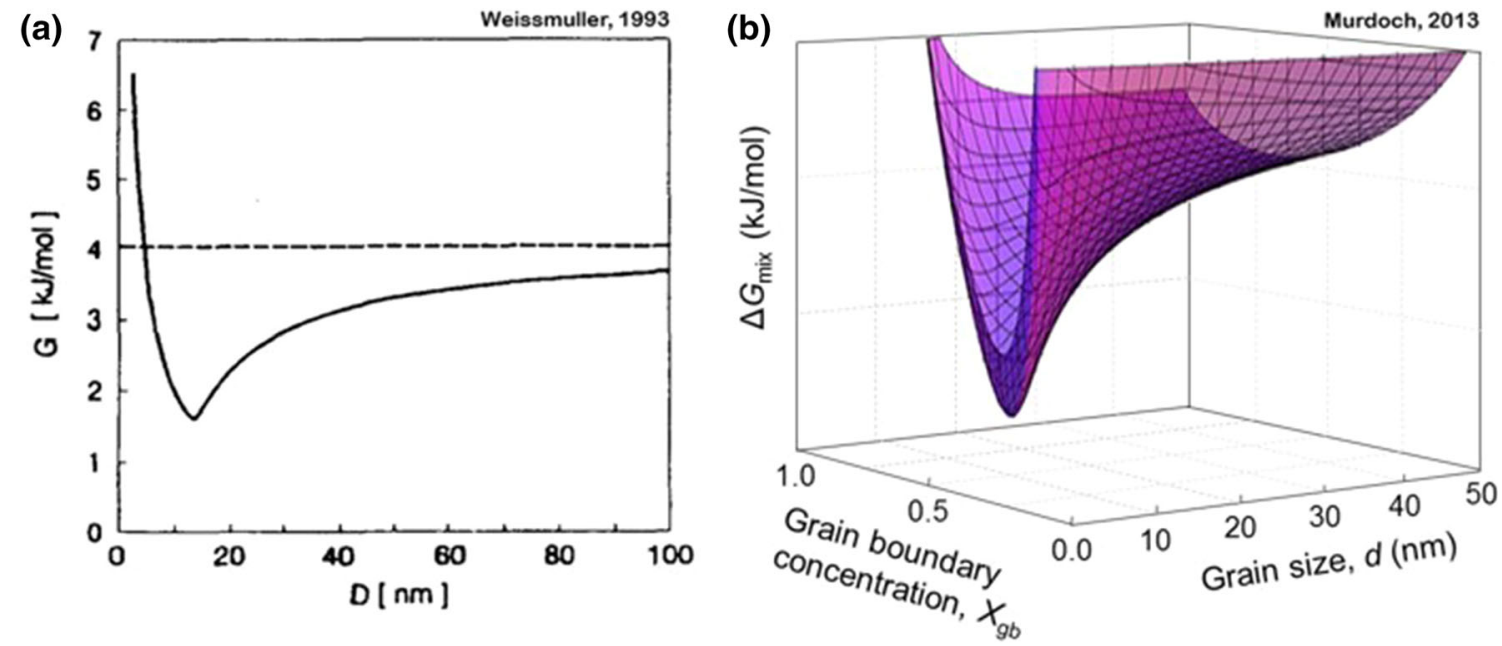

Fig. 1. (a) Solute segregation leads to a minimum in the free energy of a closed-system polycrystal at a specific grain size (from the work of Weissmüller, ${ }^{8}$ reprinted with permission of the publisher). (b) Free energy of mixing for an alloy with a minimum at a specific grain size and grain boundary concentration, using a regular nanocrystalline solution model (from the work of Murdoch et al. ${ }^{13}$ reproduced with permission of the Elsevier).

favorable enough to offset the excess free energy associated with the grain boundary, then the segregated grain boundary states would be stable against grain growth. This behavior can be derived from classical thermodynamics in accordance with the Gibbs adsorption isotherm, resulting in an expression for the grain boundary energy $\gamma$ for a dilute alloy with solute segregation at the grain boundaries:

$$
\gamma=\gamma_{0}-\Gamma_{\text {sat }}\left[\Delta H_{\text {seg }}+R T \ln \left(x_{\mathrm{c}}\right)\right]
$$

In this expression, $\gamma_{0}$ is the pure solvent grain boundary energy, which can be offset by an alloying addition with a positive enthalpy of grain boundary segregation $\Delta H_{\text {seg }}$, defined as the enthalpy required to take a single solute atom from the crystalline region and place it into the grain boundary in the dilute limit. $\Gamma_{\text {sat }}$ is the specific solute excess at the solute-saturated grain boundary, $x_{\mathrm{c}}$ is the solute concentration in the crystalline region, and $T$ is the absolute temperature. Based on this expression, Weissmüller showed that strongly segregating solute species can reduce the grain boundary energy to zero, or more specifically, it can lead to a minimum in the free energy of the alloy with respect to grain boundary area $\frac{\mathrm{d} G}{\mathrm{~d} A}=0$ at a finite grain size (Fig. 1a). ${ }^{8}$ Such a nanocrystalline state in the alloy has no driving force to change its grain boundary area when the grain size is equal to the equilibrium grain size; the nanocrystalline state is stable against grain growth because grain growth would require ejection of some solute into the grain interiors at an energy penalty.

The implications of this result are limited by the dilute solution assumption and the imposition of solute-saturated grain boundaries. To alleviate these assumptions, Trelewicz and Schuh ${ }^{12}$ used a regular solution approach to develop the regular nanocrystalline solution (RNS) model. In addition to a crystalline grain interior region, the RNS model includes a grain boundary region, defined by two variables: the grain boundary volume fraction, which in their model mapped monotonically to grain size, and the grain boundary solute concentration, which is a measure of the degree of grain boundary segregation. As in the classical regular solution model, the free energy of the alloy state is determined from the internal energy, calculated by summing the energies of the bonds (assuming a random distribution of solute in the crystalline and grain boundary regions and considering nearestneighbor pairwise interactions), and from the configurational entropy of the full system. In this way, the free energy calculation was not restricted to dilute solutions or saturated grain boundaries, thus allowing a more detailed representation of free energy as a function of a variety of structural and chemical parameters. This is shown for a specific pair of variables (grain size and grain boundary solute content) in Fig. $1 \mathrm{~b}$ and compared with a free energy plot from Weissmüller in Fig. 1a, which is presented only against a single variable (grain size). The stable state is determined by finding the minimum in free energy, which for an alloy with a nanocrystalline state stable against grain growth occurs at a finite grain size and an equilibrium solute concentration at the grain boundary (Fig. 1b). ${ }^{13}$ The Trelewicz-Schuh RNS model has been refined and extended by other authors to different more specific situations, with similar general outputs in each case. ${ }^{14,15}$

The stability of a nanocrystalline state as described in these early analytical models, and models based on these same concepts that are not detailed here, ${ }^{16-20}$ is largely determined by the enthalpy of segregation of the alloy and the energy penalty of 
the unalloyed grain boundary. Experimental studies of such thermodynamic stability have been conducted by alloying with a solute species that has a large difference in atomic radius compared with the solvent element to promote segregation driven by elastic mismatch. ${ }^{11,21-25}$ Typically, it is observed that these alloys can retain nanocrystalline states at low homologous temperatures but undergo phase separation at higher temperatures, which triggers loss in solute enrichment at grain boundary sites and results in rapid grain growth. Thus, the local minimum in free energy used to identify stable nanocrystalline alloys based on these models alone is only sufficient to claim a metastability of the segregated grain boundary state: These alloys may be stable against grain growth but not necessarily against second-phase precipitation.

Recently, some models have been used to identify hypothetical alloys in which a grain-boundary segregated solid solution is quantitatively of lower free energy than the known competing bulk states, including second phases as well as coarser grains. 9, 13,26-33 Designing for this type of stabilization requires considerations based primarily on the chemistry both in the bulk material and specifically at grain boundaries. As a result, models of thermodynamic stabilization are decidedly different from grain growth models as they are concerned with the energies of different nanocrystalline configurations as opposed to the dynamics of grain growth. In this article, we review this recent work on the thermodynamic stabilization of nanocrystalline alloys against both grain growth and phase separation. In these alloys, nanocrystallinity is expected to be preserved to higher temperatures and possess a more reliable and predictable stability due to its thermodynamic nature. Additionally, the proposed existence of nanocrystalline ground states marks a shift in how grain boundaries are considered thermodynamically.

\section{NANOCRYSTALLINITY AT EQUILIBRIUM}

Consider a nanocrystalline state at a specific grain size, $d^{*}$, corresponding to the minimum in free energy with respect to changes in grain size. If this state has a lower free energy than competing bulk phases, this state is the true equilibrium state; if such a nanostructure could be accessed, then it would be stable against grain growth and phase separation. Murdoch et al. ${ }^{9,13}$ first addressed this problem using an analytical approach based on the RNS model. In the RNS framework, a particular alloy system can be described by interaction parameters $\omega$, which are used to define the solutesolvent bond energies within the crystal (c) and grain boundary $(\mathrm{gb})$ regions:

$$
\omega_{\mathrm{c}}=E_{\mathrm{AB}}^{\mathrm{c}}-\frac{E_{\mathrm{AA}}^{\mathrm{c}}+E_{\mathrm{BB}}^{\mathrm{c}}}{2}
$$

$$
\omega_{\mathrm{gb}}=E_{\mathrm{AB}}^{\mathrm{gb}}-\frac{E_{\mathrm{AA}}^{\mathrm{gb}}+E_{\mathrm{BB}}^{\mathrm{gb}}}{2}
$$

where $E$ is the energy per bond classified by the subscript, which represents the types of species bonded $(\mathrm{A}=$ solvent and $\mathrm{B}=$ solute $)$, and the superscript, which denotes the bond type. The two interaction parameters can be related to the enthalpy of mixing and enthalpy of segregation to link model predictions of stability to actual alloys. ${ }^{12,34}$ Murdoch and Schuh simplified the problem by limiting their attention to positive enthalpy of mixing alloys, for which the crystalline interaction parameter can also be used to describe the miscibility gap. In this case, the free energy of both the nanocrystalline state and the bulk precipitation phase can be compared based largely on just the two parameters defined by Eqs. 2 and 3.

For the nanocrystalline state to be stable, it must lie below the miscibility gap on the free energy diagram. Chookajorn et al. ${ }^{9}$ developed a stability map for $\mathrm{W}$ alloys (shown in Fig. 2a) with corresponding free energy diagrams illustrating the difference between a predicted stable nanocrystalline alloy, W-Sc, and a classic bulk stable alloy, W-Ag. The map delineates the alloy pairs for which at least one nanocrystalline alloy has an energy lying below the miscibility gap free energy. This map was used to identify W-Ti as a candidate for exhibiting thermodynamic stability at $1100^{\circ} \mathrm{C}$. This system was subsequently explored with a W-20\% Ti alloy that exhibited no significant changes in grain size after annealing at $1100^{\circ} \mathrm{C}$ for 1 week. The basic premise of Chookajorn et al. ${ }^{9}$ is thus that a simple analytical model such as the RNS model can be used to rapidly screen possible alloys that may exhibit nanocrystalline ground states.

The thermodynamic accessibility of nanocrystalline states also suggests a need for a reevaluation of typical assumptions built into alloy phase diagrams. Zhou and $\mathrm{Luo}^{26}$ extended the approach of Murdoch et al. ${ }^{9,13}$ by using a CALPHAD evaluation of free energies to produce a corresponding phase diagram for Fe-Zr alloys, shown in Fig. 2b. They included nanocrystalline states computed using a regular solution model for grain boundary segregation developed by Wynblatt and Chatain. ${ }^{35}$ In this case, the segregated nanocrystalline states were less energitcally favorable than the $\mathrm{Fe}_{23} \mathrm{Zr}_{6}$ compound, and thus only metastable grain size information could be provided. In an alloy exhibiting true nanocrystalline stability, phase diagrams are expected to include phase transitions between bulk phases and nanostructured states, as well as twophase regions possessing unique nanostructural features. As the thermodynamic understanding of nanocrystalline alloys matures, it should lead to phase diagrams that explore a more extensive collection of possible nanocrystalline states. 

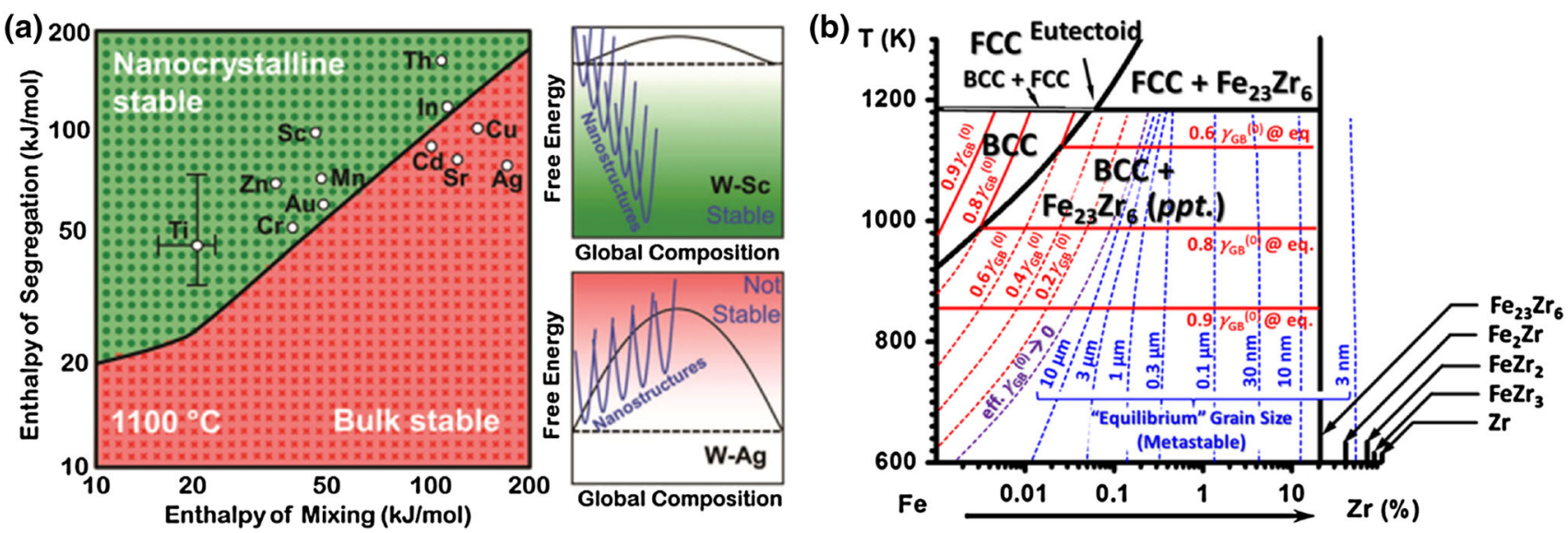

Fig. 2. (a) Stability map for W alloys based on the regular nanocrystalline solution model constructed by Chookajorn et al. ${ }^{9}$ with associated free energy diagrams, where the dashed line represents the bulk miscibility gap (reprinted with permission from AAAS). (b) A phase diagram from the work of Zhou and Luo ${ }^{26}$ of Fe-Zr system containing metastable nanocrystalline states (reproduced with permission of the Elsevier).

In bulk alloys, the configuration space to be considered in determining the equilibrium state can often be reduced to the distribution of chemical species on a lattice. However, to consider grain boundary segregation, the portion of the phase space surveyed for free energy minima must be expanded. This is a multiscale challenge: grain boundaries are disordered regions of the lattice at the atomic scale and form a complex network throughout the system at the topological, mesoscale level, as illustrated in Fig. 3. Details at these different scales are all important to a full assessment of equilibrium. At the topological level, the grain boundary network determines the equilibrium average grain size, the solute interaction with the grain boundaries, and whether nanocrystalline states are preferable to bulk phases. At the atomic scale, the local positioning of atoms in the inhomogeneous environment of the grain boundary is important for capturing segregation phenomena accurately. For example, determining the enthalpy of segregation as a function of the level of solute saturation at the grain boundary is critical to determining whether the excess grain boundary energy can be reduced to zero in a particular alloy system.

A multiscale description of the grain boundary state is difficult to capture analytically, and as such the analytical models discussed above rely on the definition of a single average grain boundary site, and the fraction of those sites in the system then becomes the core descriptor of a nanocrystalline structure. Atomistic simulations, on the other hand, have demonstrated great utility in describing the atomistic environment of the grain boundaries of nanocrystalline states $^{36}$ and the grain topology in studies of grain growth. Taking advantage of this, Millett et al. ${ }^{37-39}$ performed molecular statics and molecular dynamics (MD) studies of stability against grain growth. Using a Lennard-Jones

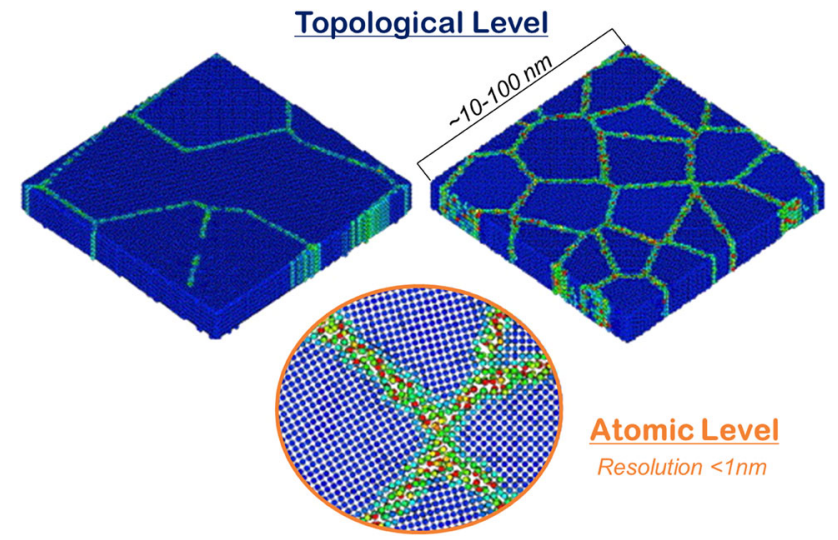

Fig. 3. Schematic of the multiscale considerations of stable nanocrystalline states on the thermodynamic phase space (images are from the work of Millett et al. ${ }^{37}$ reproduced with permission of the Elsevier).

potential, their simulations showed that placing sufficiently larger solute atoms at the grain boundaries successfully arrested grain growth by reducing the excess grain boundary energy to zero. However, from a thermodynamic perspective, two critical features are missing from such an approach for studying nanocrystalline ground states. First, grain boundary segregation should occur thermodynamically, such that the chemical potential of the alloy is constant throughout the system, which is not obeyed by artificially placing solute at the grain boundary. Second, the simulation must be sufficiently long such that bulk phases can nucleate, which is not easy to capture in an MD simulation because of the longer time scales associated with diffusion. For the time and length scales required to study the thermodynamics of nanostructured states, a statistical mechanics-based approach offers many advantages, and we turn our attention to these models in the next sections. 


\section{Thermodynamic Monte Carlo Simulations}

In statistical mechanics, the equilibrium behavior of an alloy is determined by taking thermal averages at the atomic level. For closed systems at a fixed temperature (i.e., in the canonical ensemble), the probability that a particular alloy configuration $m$ is the equilibrium state depends on the energy of the configuration $E_{\mathrm{m}}$ as $P_{\mathrm{m}}=\left(\mathrm{e}^{-\frac{E_{\mathrm{m}}}{k T}}\right) / Q$, where $\mathrm{k}$ is the Boltzmann constant and $T$ is the absolute temperature. The partition function $Q=\sum_{i} e^{\frac{E_{i}}{k T}}$ represents the size of the configuration space and can be related to thermodynamic quantities, such as entropy and free energy. Thus, if the energies of all configurations are known, then the canonical ensemble partition function and the probabilities of each configuration can be determined and used to identify the preferred state of the alloy and calculate relevant thermodynamic information.

Monte Carlo is a stochastic method for approximating the thermal averages of statistical mechanics, capturing statistical fluctuations, and connecting this information to macroscopic thermodynamic quantities. Sampling the configuration space is not a trivial task, as most configurations in the space contribute insignificantly (have very low probabilities) to the equilibrium; thus, simple sampling methods can be prohibitively inefficient. Monte Carlo simulations can be devised to instead sample configurations in the phase space at a rate corresponding to their probability of occurring in the ensemble, which is termed importance sampling. This is done by sampling the space through transitions, where a new configuration $j$ is considered for sampling by applying a transformation to the current configuration $i$. The Metropolis algorithm ${ }^{40}$ then provides stochastic rules for accepting the transition from state $i$ to $j$ by the transition probabilities:

$$
P_{i \rightarrow j}=\left\{\begin{array}{cc}
\mathrm{e}^{-\frac{E_{j}-E_{i}}{k T}}, & \text { if } E_{j}>E_{i} \\
1, & \text { otherwise }
\end{array}\right.
$$

According to this method, if the new configuration has a lower energy, then the system always transitions into it, but if it has a higher energy, then the transition only occurs with a probability determined by the energy increase and the temperature (with transitions to higher-energy states more probable at elevated temperatures). Typically, an initial configuration is chosen, and transitions are attempted and performed according to the Metropolis algorithm until the system reaches equilibrium, at which point any dependence on the choice of initial configuration has been eliminated.

When defining transitions, the goal is to explore the configuration space efficiently with respect to its degrees of freedom. For example, for bulk crystalline alloys, the alloy configuration space can be simplified to consider the distribution of solute on a lattice, as is done in the Ising model. ${ }^{41}$ In closed systems, atom swaps are used to transition through the configuration space, where a random solute atom and solvent atom from within the lattice have their lattice positions exchanged, leading to a new configuration. The nature of this transition has two important features. First, this mechanism is not meant to represent a physical process but rather to sample the configuration space without getting trapped in metastable states corresponding to local minima in the free energy, which is assisted by the long-range nature of these swaps. At the same time, a single atom swap does not produce an independent sample configuration from the phase space; it is highly dependent on the previous configuration. Therefore, to collect uncorrelated samples of the phase space and to satisfy the ergodic hypothesis, many swaps must be performed in between samples before including a new state in calculations of macroscopic thermodynamic quantities. At moderate temperatures, it has been demonstrated in a number of studies that this sampling approach successfully finds the global minimum in free energy and captures the expected phase equilibria as well as enthalpic and entropic behavior of binary alloys. $^{42-45}$

\section{MONTE CARLO SIMULATIONS CONSIDER- ING NANOCRYSTALLINE STATES}

This general Monte Carlo formulism can be adapted to include grain boundaries in the description of the configurational space of the alloy. As discussed, the presence of grain boundaries and their interaction with segregants introduces additional degrees of freedom at both the atomistic scale, related to the structure of the grain boundary, and at the mesoscale, related to the topology and crystallography of the grain boundary network. Two types of Monte Carlo simulations have been developed, each designed to focus at a particular scale, and to provide a different level of information regarding the stability of nanocrystalline states.

\section{Monte Carlo Simulations at the Atomic Level of Grain Boundaries}

A particular grain boundary can take on different equilibrium atomic configurations, known as complexions, ${ }^{46}$ with varying grain boundary thicknesses, disorder, and chemistry. As such, Monte Carlo studies of grain boundary segregation at the atomic level should consider the added positional degrees of freedom available for atoms at the grain boundary by including appropriate transition operations to reach the equilibrium grain boundary structures for a particular nanocrystalline alloy. Simulations to study grain boundary segregation in this way were developed to measure the extent of segregation at different grain boundaries and to analyze structural transitions that occur within the 
grain boundary. ${ }^{47-51}$ Such methods have been more recently adapted by Detor and $\mathrm{Schuh}^{28}$ and Purohit et al. ${ }^{29,30}$ to study grain boundary stabilization.

The transition event used to sample the phase space of the alloy must allow atoms to relax locally. A common approach is to accompany each solutesolvent atom swap with atomic relaxation of the system to maintain zero hydrostatic stress, for example by straining the system incrementally and using conjugate gradient relaxations to allow the atoms to utilize the off-lattice degrees of freedom at the grain boundary. The new, depressurized state is then considered for transition according to the Metropolis algorithm, where the energies of each state are typically calculated using many-body potentials. Such a simulation produces the lowest free energy state for the alloy, but it is constrained to the initial grain topology provided because the transition event does not create or remove grain boundary area, and the relaxations permitted do not allow for large atomic reconfiguration. Thus, to assess nanocrystalline stability, simulations at fixed grain sizes must be compared with single-crystal simulations to determine the more favorable state, as these states are not considered simultaneously in the Monte Carlo framework.

By sampling the atomic arrangements in this manner, an atomistic Monte Carlo simulation surveys the possible grain boundary structures available for a particular interface and thus provides a more detailed consideration of the free energy of grain boundary segregation. Using this method, Detor and $\mathrm{Schuh}^{28}$ showed, in a study of $\mathrm{Ni}-\mathrm{W}$ nanocrystalline alloys, that the enthalpy of segregation decreases exponentially with increasing solute content for nanocrystalline systems with grain sizes between 2 and $4 \mathrm{~nm}$, as shown in Fig. 4a. This strong dependence is an important consideration in predicting stable nanocrystalline states, where using the dilute solution enthalpy of segregation and assuming solute-saturated grain boundaries can lead to a major overestimation of the energetic benefit of segregation at higher solute concentrations, as shown in Fig. $4 \mathrm{~b}$, where the solute composition at the grain boundary according to the McLean isotherm (dashed lines) is substantially overpredicted according to the Monte Carlo simulations (solid lines) for Ni-W $\left[\Delta H_{\text {seg }}=10 \mathrm{~kJ} /-\right.$ mol (Ref. $\left.{ }^{28}\right)$ ]. To determine whether the nanocrystalline state is stable, the grain boundary energy (which is defined here as the excess enthalpy in the equilibrated nanocrystalline state compared to the equilibrated single crystal state normalized by the total grain boundary area) is calculated from simulated structures to determine whether it is less than or equal to zero. However, only the enthalpies are considered in such a calculation. Because the free energies are not known, an accurate assessment of thermodynamic stability at finite temperatures is difficult with this approach.
The shortcoming of atomistic Monte Carlo simulations for considering the stability of nanocrystalline states is that the portion of the phase space considered in a given simulation does not simultaneously include both nanocrystalline and singlecrystalline states. Moreover, to identify the stable nanocrystalline state, all possible nanocrystalline configurations at the topological level should be considered in the free energy minimization to identify the nanostructure of the equilibrated state. As a result, whereas the atomic position degrees of freedom at the grain boundary are important at the grain boundary structure level, the topological degrees of freedom of the grain boundary network must be explored as part of the Monte Carlo sample space to determine whether a nanocrystalline state exists at equilibrium and to assess the mesoscale structure of the state.

\section{Monte Carlo Simulations at the Topological Level of Grain Boundaries}

For a Monte Carlo simulation to provide information on the stability of nanocrystalline states, the sampling method should have the freedom to add or remove grain boundary area in search of the lowest free energy nanostructure. To make such a description of the configuration space more computationally feasible, it is convenient to start from a more coarse-grained approach that foregoes the atomic-level description of grain boundary structure and its dependence on local factors, such as the adjoining grain orientations, in favor of describing the effects of alloying on a larger network of average grain boundaries. Chookajorn and Schuh ${ }^{31}$ modified the Ising approach ${ }^{41-45}$ to incorporate the possibility of stable nanocrystalline states. Unlike the original Ising model, each lattice site in the alloy is not only prescribed with an occupying chemical species but also is associated with a particular grain, denoted by a grain number, such that nearest-neighbor bonds between two lattice sites with different grain numbers constitute a grain boundary, as shown in Fig. 5. Under this description, the internal energy of a particular configuration $E_{\mathrm{m}}$ can be written by summing nearest-neighbor bonds, as was done in the RNS model:

$$
\begin{aligned}
E_{\mathrm{m}}= & N_{\mathrm{AA}}^{\mathrm{c}} E_{\mathrm{AA}}^{\mathrm{c}}+N_{\mathrm{AB}}^{\mathrm{c}} E_{\mathrm{AB}}^{\mathrm{c}}+N_{\mathrm{BB}}^{\mathrm{c}} E_{\mathrm{BB}}^{\mathrm{c}}+N_{\mathrm{AA}}^{\mathrm{gb}} E_{\mathrm{AA}}^{\mathrm{gb}} \\
& +N_{\mathrm{AB}}^{\mathrm{gb}} E_{\mathrm{AB}}^{\mathrm{gb}}+N_{\mathrm{BB}}^{\mathrm{gb}} E_{\mathrm{BB}}^{\mathrm{gb}}
\end{aligned}
$$

where $E$ is the pairwise bond energy between the species specified by the subscript and with the bonding region delineated by the superscript, and $N$ is the number of such bonds. The consideration of different possible grain boundary configurations increases the size of the phase space that is explored by Monte Carlo in search of the minimum free energy state. 
(a)

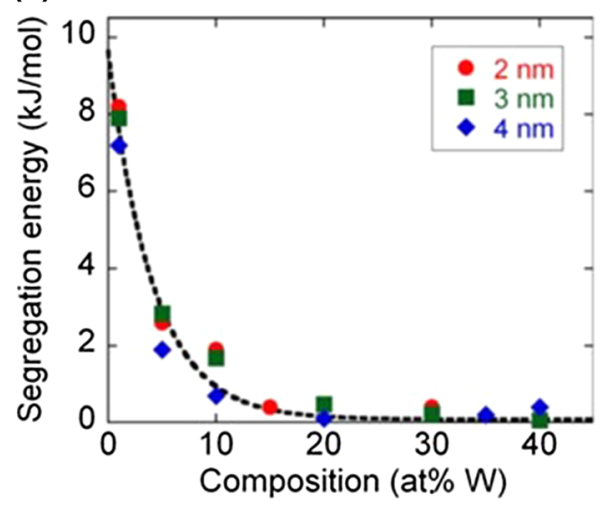

(b)

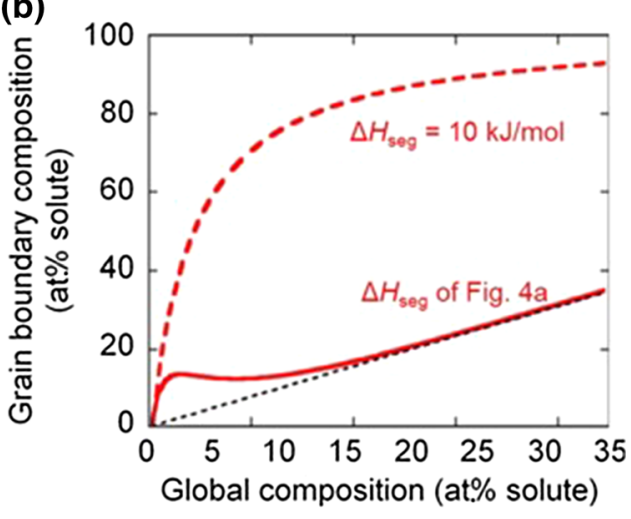

Fig. 4. (a) Reduced enthalpy of segregation with increasing solute concentration determined from atomistic-level Monte Carlo simulations by Detor and Schuh, ${ }^{28}$ which they showed is expected to drive (b) lower saturation levels at grain boundaries than predicted by analytical models using a fixed value, dilute limit enthalpy of segregation value. Images reproduced with permission of the Elsevier.

To sample configurations with different grain boundary topologies, in addition to atom swaps, two types of grain swaps were proposed by Chookajorn and Schuh: A random grain boundary site can change its grain number to that of an adjacent grain (grain boundary motion) or to an entirely new grain number (nucleation of a new grain). This evolution of the grain topology is based on the classical Potts model $^{52}$ and is likewise vulnerable to grain faceting and pinning, ${ }^{53}$ which increases the likelihood of being trapped in a local energy minimum and falsely identifying a stable nanocrystalline state. To address this, the temperature can be decreased slowly at the beginning of each simulation to facilitate grain boundary motion during the early stage.

In the cases where stable nanostructures are not thermodynamically the most favorable state, this model replicates bulk alloy thermodynamics that are consistent with the Ising model. For alloys that favor grain boundary segregation, however, the Monte Carlo method provides results that are consistent with the analytical models introduced earlier: Nanocrystalline states emerge as preferred structures. The model also uncovers unique behaviors that arise from the synchronicity between the equilibration of both grain and chemical structures, which are not predicted by regular solution models, and to this point, remain unique predictions of the Monte Carlo model.

To probe systematically the different types of stable nanostructures that can be found by the simulation, one can use a perspective based on the bond energies available to the model, which can be related using the alloy interaction parameters (cf. Eqs. 2 and 3). These two interaction energies along with the grain boundary energy penalty of the unalloyed components determine the relative preference for different bond environments and can be used to estimate the enthalpically preferred structural configuration. A stability map outlining several behavioral trends on the interaction parameter space was proposed by Chookajorn and Schuh ${ }^{31}$ to

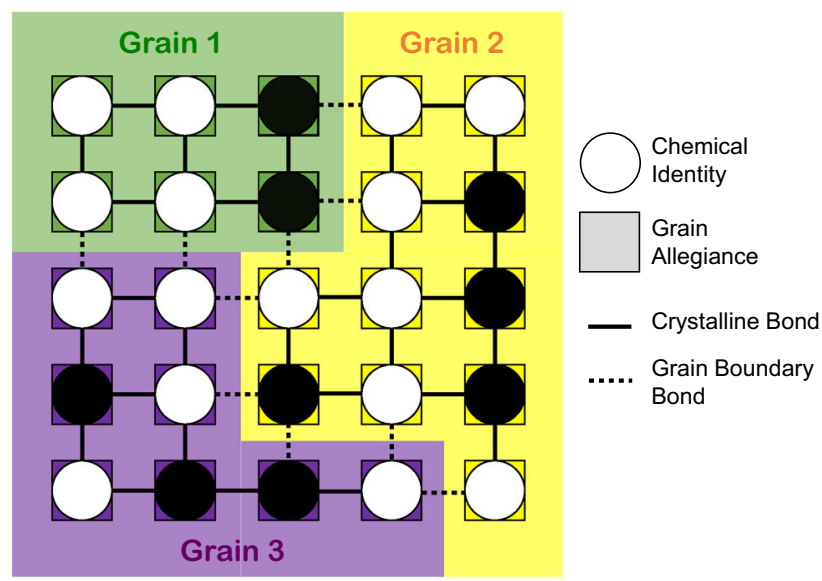

Fig. 5. A schematic of the lattice-based nanocrystalline alloy model developed by Chookajorn and Schuh, ${ }^{31}$ where each lattice site contains chemical and grain allegiance information.

provide guidelines of regions in which distinct types of nanostructures are expected. Such a map is shown in Fig. 6, including the positive interaction energy quadrant originally developed in Ref. ${ }^{31}$ and expanded here to include the other three quadrants, as described in more detail next.

In Fig. 6, the bulk structure (red region, Fig. 6b), and segregated nanocrystalline structure (green region, Fig. 6e) regions are the classic states predicted by the RNS model and are similar to the RNS-based map regions plotted on different axes in Fig. 2a. However, the simulations are not limited to studying fully segregated grain boundary states and bulk phases. Duplex nanostructures (blue region, Fig. 6d) exhibit simultaneous solute segregation and precipitation, and phase-separated polycrystals (yellow region, Fig. 6c) do not exhibit segregation but are characterized by precipitates as well as grain boundaries. The latter two nanostructures, which have solute precipitates as well as grain boundaries at equilibrium, are not the minimum 


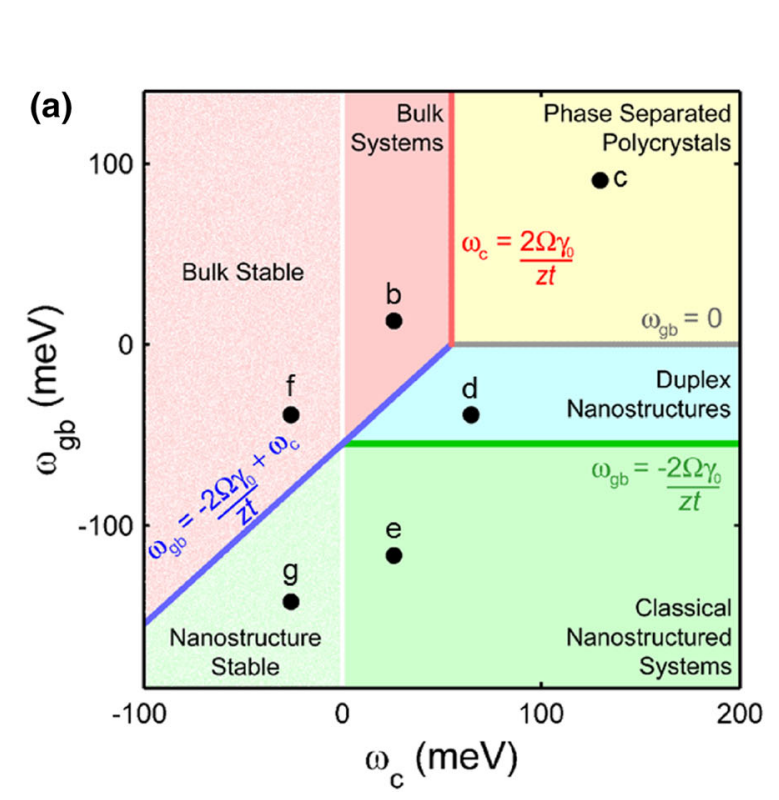

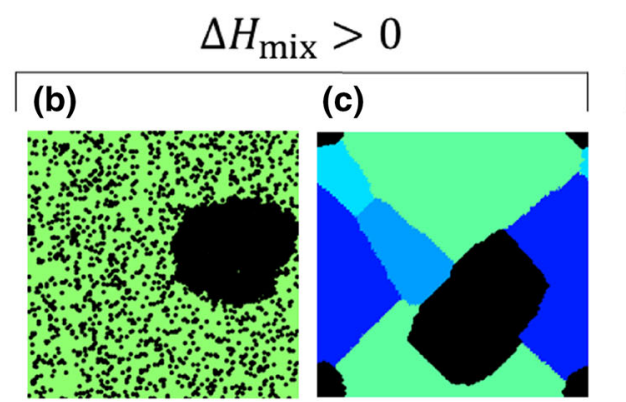

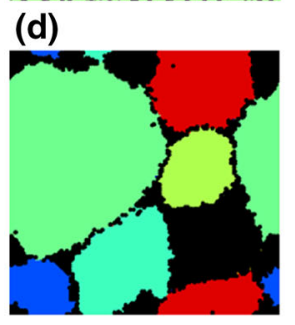

(e)

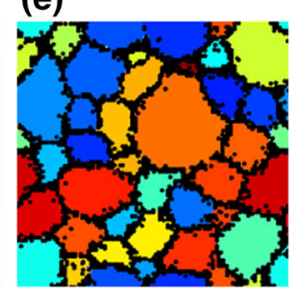

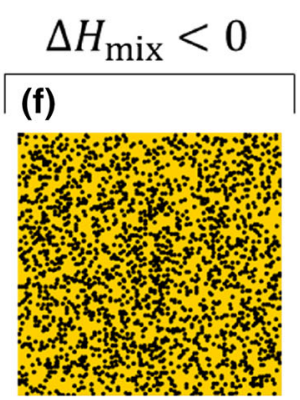

(g)

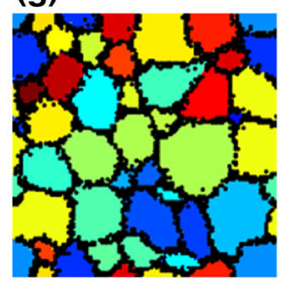

Fig. 6. (a) Stability map of six general regions of nanocrystalline stability: (b) bulk, single-crystalline alloy with positive enthalpy of mixing; (c) phase-separated polycrystal with undoped grain boundaries; (d) duplex nanostructured states with segregated grain boundaries; (e) segregated nanocrystalline state with positive enthalpy of mixing; (f) bulk single-crystalline alloy with negative enthalpy of mixing, and (g) segregated nanocrystalline state with negative enthalpy of mixing.

internal energy configurations possible; in both cases, the lowest internal energy state would be a single crystal, precipitated state. However, in the duplex and phase-separated polycrystal regions of the stability map, grain boundary states exist with internal energies that are in between the precipitated and disordered single-crystal states, and as such at intermediate temperatures the higher entropy available in duplex nanostructures and phase-separated polycrystals leads to a minimum free energy polycrystalline state. The ability of the lattice-based simulation to predict the stability of a wide range of structures makes it a valuable tool for alloy design, and it has been shown to compare well with some limited experimental studies of W-Ti (segregated nanocrystal) ${ }^{32}$ and W-Cr (duplex nanostructure). ${ }^{33}$

Monte Carlo simulations of this kind provide valuable thermodynamic insight as they can capture the entropy of the different equilibrium states, which is useful for constructing phase diagrams of alloys accounting for potential nanocrystalline stability. Using this method, Chookajorn et al. constructed the phase diagram of an alloy from the duplex region of the stability map, which is illustrated in Fig. $7 .^{33}$ For a comparison, simulations were conducted with an artificially imposed single-crystal state (Fig. 7a) as well as using the full grain-evolving model as described above (Fig. 7b). When the system was constrained against the formation of grain boundary states, this positive enthalpy of mixing alloy was verified to exhibit bulk precipitation at low temperatures, which evolved to a solid solution at high temperatures (Fig. 7a). However, when nanocrystalline states were allowed to evolve, at intermediate temperatures a duplex nanostructured phase emerged, which disordered into a segregated nanocrystalline state at higher temperatures (Fig. 7b). The existence of an intermediate energy level associated with the grain boundary state leads to an interesting new phase diagram that includes nanostructured states at equilibrium.

Stability maps of the kind described by Chookajorn et al. ${ }^{9,13,31}$ have only been published for positive enthalpy of mixing alloys. When using the RNS model, this is convenient as the miscibility gap can be also calculated as a function of the enthalpy of mixing, which is not necessarily the case in negative enthalpy of mixing alloys. In principle, Monte Carlo simulations can capture ordering effects more relevant to negative enthalpy of mixing systems, albeit only at compositions near the stoichiometry of the ordered phase when pairwise bond descriptions are used. Monte Carlo methods can thus provide a natural pathway to the extension of nanostructure design maps to a broader range of alloys. Figure 6 extends previously published maps to include the other quadrants outside of the positive enthalpy of mixing range that has been explored in prior work. The lines separating the regimes of different structures in quadrants II, III, and IV of this plot are derived in the same manner as proposed by Chookajorn and Schuh, ${ }^{31}$ by merely examining the enthalpic preference on the basis of the lowest bond energies. Figure $6 f$ and $g$ shows a series of sample structures from negative enthalpy of mixing regions of Fig. 6a, calculated using the Chookajorn-Schuh Monte Carlo algorithm. The negative enthalpy of 


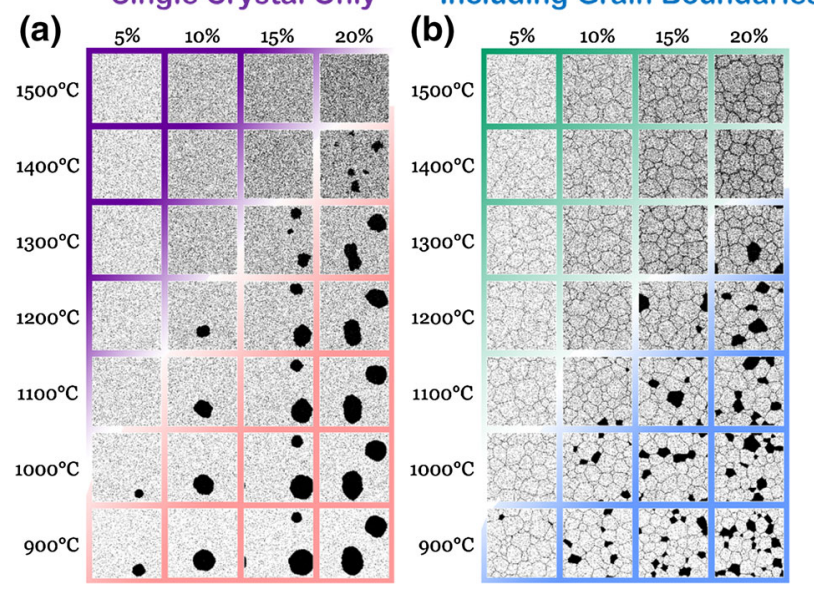

Fig. 7. Binary alloy phase diagrams constructed using a mesoscale Monte Carlo simulation: (a) assuming no nanocrystalline states are stable and (b) considering the possibility of equilibrium nanocrystalline states in the Monte Carlo simulation (from the work of Chookajorn et al. ${ }^{33}$ reproduced with permission of the Cambridge University Press).

mixing notwithstanding, the simulated structures resemble those produced in the same region using analogous positive enthalpy of mixing alloy parameters.

The analysis used to construct the stability map assumes that the formation enthalpy of the ordered compound depends only on the pairwise bond energies provided in the model description, which is too restrictive to identify stable nanostructured phases that involve ordered compounds. Because many alloys are based on negative enthalpy of mixing pairs, clearly more nuanced treatments should be developed to add detail to these regions of the stability maps. With the addition of methods that better capture ordered compounds in the Monte Carlo simulations, these regions might indeed prove to contain additional nanostructured phases in which both ordering and grain boundary segregation are at play.

\section{CHALLENGES AND OPPORTUNITIES}

As demonstrated by lattice Monte Carlo results such as those described previously, considering the topological degrees of freedom of the grain boundary network in the thermodynamic assessment of alloys allows the simulation to determine whether grain boundaries should exist in equilibrium or not, as part of its free energy minimization. The implementation used in the published studies so far has proven suitable to identifying stable nanostructures of some particular alloy systems at finite temperatures, but it does rely on local grain swap events; local events such as these are more likely to be susceptible to trapping in metastable states, which would become more acute as the temperature of interest is lowered. Local trapping would also prohibit the ergodic sampling of the ensemble at a fixed temperature, to assess quantitatively macroscopic thermodynamic quantities such as free energy and entropy. A much larger correlation time is associated with the sampling of the topology than with the atom swaps in sampling the chemical distribution, if the topology is only probed via processes of local grain boundary motion. Improving this method for sampling nanostructures such that the grain topology is not strongly correlated between samples would present an important step for conducting Monte Carlo calculations of the free energy of nanocrystalline states and for assessing their stability at lower temperatures.

The effective use of any coarse-grained model of nanocrystalline stability, including, e.g., analytical RNS-type models or mesoscale Monte Carlo simulations, for the purpose of screening of candidate nanostructured alloy systems also requires accurate estimation of "average" or "effective" energetic parameters describing grain boundary segregation and ordering interactions. The enthalpy of grain boundary segregation is not well known for most alloy systems, and therefore it is the limiting input parameter for studying a wide range of materials, with Miedema-type models and elaborate experimental techniques that measure the concentration at grain boundaries as the predominant methods. Instead, atomic-level Monte Carlo simulations at a variety of "typical" grain boundaries could be used to estimate these enthalpies.

\section{SUMMARY}

Preferential segregation of solute to grain boundaries can reduce their excess free energy, and in certain alloy systems it can, in principle, entirely relieve the energetic penalty associated with the boundaries. Such an alloy possesses a minimum in its free energy of mixing at a finite grain size, and if this minimum energy is lower than that of the competing phases, then the nanostructured state is the equilibrium state of the alloy and is stable against both grain growth and second phase formation.

The possibility of thermodynamically stable nanocrystalline states expands the portion of the phase space that should be considered in the calculation of macroscopic thermodynamic properties, both at the atomic level, where atoms are freer to rearrange locally in grain boundaries, and at the topological level, where different grain size distributions can exist. Whereas analytical models such as the regular nanocrystalline solution model and its variants provide a first-order approach to capturing grain boundary states, they rely on assumptions at each of these two scales. On the other hand, Monte Carlo simulations can be targeted at each of these different scales to address some of the open interesting questions in nanostructure stabilization. Simulations at the atomistic level can capture precise grain boundary structures, for example revealing the functional form by which 
the enthalpy of segregation decreases with increasing solute, or the influence of grain boundary crystallography on segregation behavior. However, such local atomistic models are not currently able to discern whether a single crystal or a nanocrystalline structure is the true equilibrium state due to the difficulty of calculating the free energy of each equilibrated structure. On the other hand, at the mesoscale it is possible to incorporate topological degrees of freedom in a lattice Monte Carlo simulation, so that both the nanocrystalline state and the ground state are simultaneously considered in minimizing the free energy. While such methods so far are not amenable to precise calculation of the free energy, the lowest free energy structure is determined intrinsically within the simulation.

One important direction for future work that calls for more effort on computer simulation is the interesting set of structures in which multiple bond configurations are energetically similar, and as a result, two-phase regions and phase transitions containing equilibrium nanostructures are expected. For example, "duplex nanostructures" are those in which the ground state is proposed to be precipitation, but the first excited state of the system as temperature is raised is the decoration of grain boundaries by solute. When the alloy space is broadened to accommodate further alternative configurations, e.g., ordered compound formation in negative enthalpy of mixing systems, a variety of nontrivial nanostructured states might be possible.

\section{ACKNOWLEDGEMENT}

This work was supported by the U.S. Army Research Office under grant W911NF-14-1-0539 and through the Institute for Soldier Nanotechnologies at MIT. A.K. acknowledges a National Science Foundation Graduate Research Fellowship.

\section{REFERENCES}

1. K. Lücke and K. Detert, Acta Metall. 5, 628 (1957).

2. J.W. Cahn, Acta Metall. 10, 789 (1962).

3. A. Michels, C.E. Krill, H. Ehrhardt, R. Birringer, and D.T. Wu, Acta Mater. 47, 2143 (1999).

4. M. Hillert, Acta Metall. 36, 3177 (1988).

5. K. Boylan, D. Ostrander, U. Erb, G. Palumbo, and K.T. Aust, Scripta Metall. Mater. 25, 2711 (1991).

6. E.D. Hondros, Proc. R. Soc. A 286, 479 (1965).

7. J.W. Cahn, Surface Segregation in Metals and Alloys, ed. by W.C. Johnson, and J.M. Blakely (Metals Park, ASM, 1979), p. 3.

8. J. Weissmüller, Nanostruct. Mater. 3, 261 (1993).

9. T. Chookajorn, H.A. Murdoch, and C.A. Schuh, Science 337, 951 (2012).

10. K.A. Darling, B.K. VanLeeuwen, J.E. Semones, C.C. Koch, R.O. Scattergood, L.J. Kecskes, and S.N. Mathaudhu, $M a-$ ter. Sci. Eng. A 528, 4365 (2011).

11. P. Choi, M. da Silva, U. Klement, T. Al-Kassab, and R. Kirchheim, Acta Mater. 53, 4473 (2005).
12. J.R. Trelewicz and C.A. Schuh, Phys. Rev. B 79, 094112 (2009).

13. H.A. Murdoch and C.A. Schuh, Acta Mater. 61, 2121-2132 (2013).

14. M. Saber, H. Kotan, C.C. Koch, and R.O. Scattergood, J. Appl. Phys. 113, 063515 (2013).

15. M. Saber, H. Kotan, C.C. Koch, and R.O. Scattergood, J. Appl. Phys. 114, 103510 (2013).

16. R. Kirchheim, Acta Mater. 50, 413 (2002).

17. F. Liu and R. Kirchheim, Scripta Mater. 51, 521 (2004).

18. F. Liu and R. Kirchheim, J. Cryst. Growth 264, 385 (2004).

19. A. Kirchner and B. Kieback, Scripta Mater. 64, 406 (2011).

20. M. Saber, C.C. Koch, and R.O. Scattergood, Mater. Res. Lett. 3, 37 (2015).

21. P. Choi, T. Al-Kassab, F. Gärtner, H. Kreye, and R. Kirchheim, Mater. Sci. Eng. A 353, 74 (2003).

22. S.C. Mehta, D.A. Smith, and U. Erb, Mater. Sci. Eng. A 204, 227 (1995).

23. T. Hentschel, D. Isheim, R. Kirchheim, F. Müller, and H. Kreye, Acta Mater. 48, 933 (2000).

24. K.A. Darling, B.K. VanLeeuwen, C.C. Koch, and R.O. Scattergood, Mater. Sci. Eng. A 527, 3572 (2010).

25. A.J. Detor and C.A. Schuh, Acta Mater. 55, 371-379 (2007).

26. N. Zhou and J. Luo, Mater. Lett. 115, 268 (2014).

27. A.J. Detor and C.A. Schuh, J. Mater. Res. 22, 3233 (2007).

28. A.J. Detor and C.A. Schuh, Acta Mater. 55, 4221 (2007).

29. Y. Purohit, L. Sun, D.L. Irving, R.O. Scattergood, and D.W. Brenner, Mater. Sci. Eng. A 527, 1769 (2010).

30. Y. Purohit, L. Sun, D.L. Irving, C.W. Padgett, R.O. Scattergood, and D.W. Brenner, Mater. Sci. Eng. A 493, 97 (2008).

31. T. Chookajorn and C.A. Schuh, Phys. Rev. B 89, 064102 (2014).

32. T. Chookajorn and C.A. Schuh, Acta Mater. 73, 128 (2014).

33. T. Chookajorn, M. Park, and C.A. Schuh, J. Mater. Res. 30, 151 (2015).

34. H.A. Murdoch and C.A. Schuh, J. Mater. Res. 28, 2154 (2013).

35. P. Wynblatt and D. Chatain, Metall. Mater. Trans. A 37A, 2595 (2006).

36. D. Wolf, V. Yamakov, S.R. Phillpot, A. Mukherjee, and H. Gleiter, Acta Mater. 53, 1 (2005).

37. P.C. Millett, R.P. Selvam, and A. Saxena, Acta Mater. 55, 2329 (2007).

38. P.C. Millett, R.P. Selvam, S. Bansal, and A. Saxena, Acta Mater. 53, 3671 (2005).

39. P.C. Millett, R.P. Selvam, and A. Saxena, Acta Mater. 54, 297 (2006).

40. N. Metropolis, A.W. Rosenbluth, M.N. Rosenbluth, A.H. Teller, and E. Teller, J. Chem. Phys. 21, 1087 (1953).

41. G. Ceder, Comput. Mater. Sci. 1, 144 (1993).

42. L. Guttman, J. Chem. Phys. 34, 1024 (1961).

43. K. Binder, Phys. Rev. Lett. 45, 811 (1980).

44. K. Binder, $Z$ Phys. B 45, 61 (1981).

45. K. Binder, J.L. Lebowitz, M.K. Phani, and M.H. Kalos, Acta Metall. 29, 1655 (1981).

46. S.J. Dillon, M. Tang, W.C. Carter, and M.P. Harmer, Acta Mater. 55, 6208 (2007).

47. J.D. Rittner, S.M. Foiles, and D.N. Seidman, Phys. Rev. B 50, 12004 (1994).

48. S.M. Foiles, Phys. Rev. B 40, 11502 (1989).

49. D.N. Seidman, Annu. Rev. Mater. Res. 32, 235 (2002).

50. D. Udler and D.N. Seidman, Interface Sci. 6, 259 (1998).

51. D. Udler and D.N. Seidman, Acta Metall. 46, 1221 (1998).

52. M.P. Anderson, D.J. Srolovitz, G.S. Grest, and P.S. Sahni, Acta Metall. 32, 783 (1984).

53. E.A. Holm and C.C. Battaile, JOM 53, 20 (2001). 